\title{
Glycerol as a green solvent for high product yields and selectivities
}

\author{
Adi Wolfson • Christina Dlugy • Yoram Shotland
}

Received: 18 April 2006 / Accepted: 17 July 2006 / Published online: 15 August 2006

(C) Springer-Verlag 2006

\begin{abstract}
Organic synthesis is usually performed in solution to dissolve both reactants and catalysts and to deliver heat. Here, we show that glycerol, which is a non-toxic, biodegradable, and recyclable liquid manufactured from renewable sources, has a high potential to serve as alternative green solvent for organic reactions. Several catalytic and non-catalytic reactions were successfully performed in glycerol. High products yields and selectivities were achieved. Besides solubility of the reactants and the catalysts and easy separation of the product, glycerol offers several other benefits such as catalyst recycling, microwave assisting reaction, and biphasic and emulsion modes.
\end{abstract}

Keywords Green chemistry - Alternative solvents · Glycerol · Catalysis

\section{Introduction}

Solvents are used daily in numerous industrial processes as reaction medium, in separation procedures, and as diluters. As reaction medium, solvent are employed to bring reactants and/or catalysts together and to deliver heat and momentum (Reichardt 1979). In addition, the solvent may also affect activity and selectivity. The choice of the solvent, i.e., its chemical, physical, and biological nature, also plays a key role from environmental, economic, safety, handling, and products isolation point of views.

A. Wolfson $(\varangle) \cdot$ C. Dlugy $\cdot$ Y. Shotland

Chemical Engineering Department, Sami Shamoon College of

Engineering,

Bialik/Basel Sts., Beer-Sheva 84100, Israel

e-mail: adiw@sce.ac.il

Tel.: +972-86475731

Fax: +972-86475636
Water is the first solvent of choice regarding the aforementioned considerations, yet the negligible solubility of many organic and organo-metallic compounds in water limits its applications. Using organic, petroleum-based, solvents that allow dissolving a large variety of solid, liquids, and gases is usually accompanied with air, water, and land contamination. Fluorous phases (Horváth and Rábai 1994) and ionic liquids (Wasserscheid and Welton 2003) have been reported as recyclable environmentally benign reaction media. However, not only that ionic liquids and perfluorinated solvents are non-biodegradable and toxic, their production is also associated with use of high amounts of hazardous and volatile organic solvents. Supercritical fluids and especially supercritical $\mathrm{CO}_{2}$ have also been reported as green solvents, but their high critical properties still limits their practical use (Jessop and Leitner 1999).

Glycerol is usually produced as a byproduct of the transesterification of a triglyceride in the production of natural fatty acid derivatives. These derivatives are utilized in many areas from pharmaceuticals and food industry to alternative fuels, e.g., biodiesel, and thus as the production of glycerol raises its price decreases. In addition, glycerol has also promising physical and chemical properties. It has a very high boiling point and negligible vapor pressure; it is compatible with most organic and inorganic compounds, and does not require special handling or storage. Glycerol, as other polar organic solvents such as DMSO and DMF, allows the dissolution of inorganic salts, acids, and bases, as well as enzymes and transition metal complexes (TMCs), but it also dissolves organic compounds that are poorly miscible in water and is non-hazardous. Different hydrophobic solvents such as ethers and hydrocarbons which are immiscible in glycerol allow removing the products by simple extraction. Distillation of products is also feasible due to the high boiling point of glycerol. 
The purpose of this study is to explore the scope and limitations of glycerol as alternative green reaction medium. Glycerol, which is a non-toxic, biodegradable, and recyclable liquid manufactured from renewable sources, shows similar properties as an ionic liquid and has a high potential to serve as green solvent for organic syntheses. This has led us to study its possible use as such in a variety of ways. Several non-catalytic and catalytic reactions using homogeneous and heterogeneous chemo- and bio-catalysts have been thus studied in glycerol. The unique physico-chemical nature of glycerol enables easy separation of the product by extraction or distillation together with catalyst recycling. These properties can also be translated into other processes which require non-aqueous polar solvents such as non-aqueous emulsions (Imhof and Pine 1997), as well as applications in microwavepromoted synthesis (Kappe 2004).

\section{Experimental}

Several catalytic and non-catalytic reactions were performed in glycerol as illustrate in Fig. 1. The amounts of substrates are also noted in the figure. All chemicals were purchased from Sigma-Aldrich except glycerol that was purchased from Frutarom Ltd. (99\% purity).

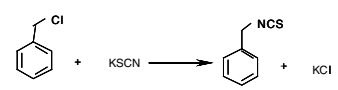

$0.2 \mathrm{~g} \quad 0.15 \mathrm{~g}$

a. Nucleophilic substitution

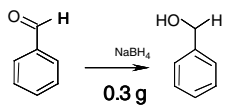

$1 \mathrm{~g}$

b. Reduction

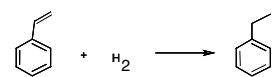

$0.53 \mathrm{~g} 20 \mathrm{~atm}$

c. Catalytic reduction

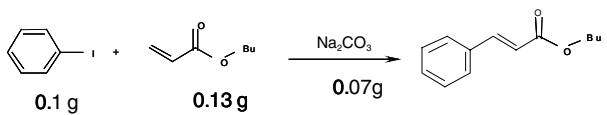

d. Heck coupling

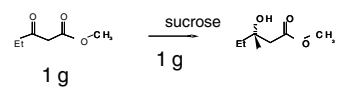

e. Asymmetric hydrogenation

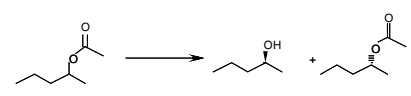

$0.1 \mathrm{~g}$ (racemate)

f. Transesterification
In a typical reaction, $10 \mathrm{~mL}$ of glycerol was heated in an oil bath to the required temperature $\left(80^{\circ} \mathrm{C}\right)$. Then, the substrates were added and the reaction mixture was mixed for the corresponding time. At the end of the reaction, the glycerol phase was cooled down and the products were separated by extraction with $3 \times 10 \mathrm{~mL}$ of diethyl ether or ethyl acetate.

The catalytic reduction of styrene and the Heck coupling of iodobenzene and butyl acrylate (reactions c and d) were initiated by dissolving the homogeneous complex or dispersing the heterogeneous catalyst in glycerol before addition of the substrates. When transition metal complexes were employed as catalysts rhodium chloride or palladium acetate were mixed with the water-soluble ligand tris-(3-sulfophenyl)-phosphine trisodium salt (TPPTS), yielding $\mathrm{Rh}(\mathrm{TPPTS})_{3} \mathrm{Cl}_{2}$ (1) and $\mathrm{Pd}(\mathrm{TPPTS})_{2}(\mathrm{Ac})_{2}$ (2) correspondingly.

For the catalytic reduction of styrene, the catalyst was dissolved in glycerol in a home-made $20 \mathrm{~mL}$ stainless steel reactor followed by the addition of styrene and Pluronic (PE 6400 BASF), when needed. Then the reactor was pressurized with molecular hydrogen to 10 bar and heated to $80^{\circ} \mathrm{C}$.

The microwave-assisted palladium-catalyzed Heck coupling was conducted in an unmodified microwave (900 W). The catalyst was first dissolved in $10 \mathrm{~mL}$ glycerol followed by the addition of both iodobenzene and butyl acrylate to a $50 \mathrm{~mL}$ vessel that was closed with watch glass. Then the reaction mixture was heated in the microwave oven for $5 \mathrm{~min}$ from room temperature to $80^{\circ} \mathrm{C}$, cooled down to room temperature and extracted with diethyl ether.

The asymmetric reduction of ethyl acetoacetate was performed with both free baker's yeasts (FBY) and immobilized baker's yeasts (IBY) in glycerol: $10 \mathrm{~g}$ of FBY (Sigma, type II) or $50 \mathrm{~g}$ of IBY (prepared from $10 \mathrm{~g}$ of FBY as previously described in literature (Howarth 2001)) were added to a mixture of $50 \mathrm{~mL}$ of glycerol in a $250 \mathrm{~mL}$ bottle and shaken for $30 \mathrm{~min}$. Then $5 \mathrm{~g}$ of sucrose were added and the bottle was shaken for extra $10 \mathrm{~min}$ before $1 \mathrm{~g}$ of ethyl acetoacetate was added. Finally, the bottle was shaken at $300 \mathrm{rpm}$ for $48 \mathrm{~h}$ at $37^{\circ} \mathrm{C}$. At the end of the reaction, the product was extracted with diethyl ether $(3 \times 50 \mathrm{~mL})$.

The products were analyzed by GC using HP-1 column to determine the conversion of the reaction, while enantiomeric excess was detected by GC analysis by using Astec Chiraldex G-TA chiral column.

\section{Results and discussion}

Glycerol as alternative green reaction medium

As previously mentioned, the environmental impact of a solvent is derived from its physical, chemical, and biological 
Table 1 Comparison of the different properties of alternative green solvents

\begin{tabular}{|c|c|c|c|c|}
\hline & Glycerol & $\mathrm{H}_{2} \mathrm{O}$ & $\mathrm{BmimPF}_{6}$ & $\mathrm{C}_{6} \mathrm{~F}_{14}$ \\
\hline $\mathrm{NBP}\left({ }^{\circ} \mathrm{C}\right)$ & 290 & 100 & $>300$ & $58-60$ \\
\hline Vapor pressure at $50^{\circ} \mathrm{C}(\mathrm{mmHg})$ & $<1$ & 92.51 & $<1$ & n.a \\
\hline Dielectric constant $\left(25^{\circ} \mathrm{C}\right)$ & 42.5 & 78.5 & 11.4 & $<5$ \\
\hline Viscosity $(\mathrm{cP})\left(30^{\circ} \mathrm{C}\right)$ & 629 & 1 & 312 & n.a \\
\hline Density $(\mathrm{g} / \mathrm{mL})$ & 1.29 & 1 & 1.37 & 1.66 \\
\hline Biodegradab-ility & Yes & - & No & No \\
\hline $\mathrm{LD}_{50}$ (Oral-Rat) (mg/kg) & 12,600 & $>90,000$ & $\sim 1500$ & $\sim 5000$ \\
\hline TMCs modification & Without/minor & Minor & Without & Tedious \\
\hline
\end{tabular}

properties. Hence, the properties of glycerol were compared with those of water, 1-butyl-1-methylimidazolium hexafluorophospate $\left(\mathrm{BmimPF}_{6}\right)$, and perfluorohexane $\left(\mathrm{C}_{6} \mathrm{H}_{14}\right)$ as representatives of ionic liquid and fluorous solvent (Table 1). All four solvents are recyclable, yet only glycerol and water are biodegradable and renewable. The low vapor pressure of glycerol and $\mathrm{BmimPF}_{6}$ is another environmental advantage. Glycerol is also, clearly, the less toxic solvent among the three organic solvents as express by its high $\mathrm{LD}_{50}$.

The physical-chemical nature of the solvent also dictates its suitability as reaction medium. The polarity of the solvent will control the solubility of organic, inorganic, and organo-metallic compound within. Water, which is the most polar solvent in the world, easily dissolve acids, bases, and salts; however, most organic and organo-metallic molecules have negligible solubility in water. Glycerol and $\mathrm{BmimPF}_{6}$ are also relatively polar and can dissolve inorganic and organic compounds. However, their polarity makes them immiscible in a variety of hydrophobic solvents and thus allows products separation by simple extraction. The high viscos- ity of glycerol and $\mathrm{BmimPF}_{6}$ might be a disadvantage, yet increasing the temperature above $50^{\circ} \mathrm{C}$ decreases their viscosity. Finally, while perfluorohexane requires tedious modification of organo-metallic complexes, all the other solvent usually dissolve TMCs with simple modification.

\section{Organic synthesis in glycerol}

The experimental data accumulated so far in a wide scope of organic syntheses in glycerol proves our assumption that glycerol can perform many of the same functions as organic petroleum-based solvents and ionic liquids, as shown in Table 2. Different organic transformations involve both organic and inorganic compounds (March 1992) and are frequently performed in highly polar hazardous organic solvents, e.g., DMF, acetonitrile, and DMSO, or in waterorganic biphasic systems. By comparison, our laboratory experience has shown that performing the nucleophilic substitution of benzyl chloride with potassium thiocyanate in
Table 2 Organic synthesis in glycerol $^{\mathrm{a}}$

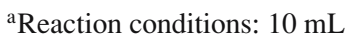
glycerol, $80^{\circ} \mathrm{C}$. Separation of the products was done by extraction with $3^{*} 10 \mathrm{~mL}$ of diethyl ether or ethyl acetate.

${ }^{\mathrm{b}}$ Enantiomeric excess.

${ }^{\mathrm{c}} 50 \mathrm{~mL}$ glycerol, $34^{\circ} \mathrm{C}$. Separation of the products was done by extraction with $120 \mathrm{~mL}$ of diethyl ether in three sequential extractions of $40 \mathrm{~mL}$.
}

\begin{tabular}{|c|c|c|c|c|}
\hline Entry & Reaction & Catalyst (g) & $t(\mathrm{~h})$ & $\begin{array}{l}\text { Conversion } \\
(\%)[\text { ee }(\%)]^{\mathrm{b}}\end{array}$ \\
\hline \multicolumn{5}{|c|}{ Non-catalytic } \\
\hline 1 & Nucleophilic substitution & - & 5 & 95 \\
\hline 2 & Reduction & - & 0.25 & 100 \\
\hline \multicolumn{5}{|c|}{$\begin{array}{l}\text { Homogeneous and } \\
\text { heterogeneous } \\
\text { chemo-catalysis }\end{array}$} \\
\hline \multirow[t]{3}{*}{3} & Catalytic reduction & $\mathbf{1}(0.095)$ & 3 & 61 \\
\hline & & $\begin{array}{l}\mathbf{1}(0.095)+\text { Pluronic } \\
(0.1)\end{array}$ & 3 & 84 \\
\hline & & $5 \% \mathrm{Pd} / \mathrm{C}(0.106)$ & 3 & 100 \\
\hline \multirow[t]{3}{*}{4} & Heck coupling & $2(0.0161)$ & 4 & 100 \\
\hline & & $\begin{array}{l}2 \text { (0.0161) } \\
\quad \text { (microwave) }\end{array}$ & 4 & 56 \\
\hline & & $5 \% \mathrm{Pd} / \mathrm{C}(0.02)$ & 0.08 & 78 \\
\hline \multicolumn{5}{|c|}{$\begin{array}{l}\text { Homogeneous and } \\
\text { heterogeneous } \\
\text { bio-catalysis }\end{array}$} \\
\hline \multirow[t]{2}{*}{5} & Asymmetric reduction ${ }^{c}$ & FBY (10) & 48 & $74(99)$ \\
\hline & Asymmetric reduction $^{c}$ & IBY (10) & 48 & $100(99)$ \\
\hline 6 & Trans-esterification & Novozyme-435 ( 0.1$)$ & 1.5 & $60(99)$ \\
\hline
\end{tabular}


glycerol (Table 2, entry 1), e.g., allowed dissolving both substrates in one phase and resulted in high conversion to benzyl thiocyanate.

The reduction of organic compounds is a fundamental transformation in organic synthesis. Metal hydrides are often used as stoichiometric reducing agent due to their relatively low price and to avoid high hydrogen pressure. Sodium borohydride can selectively reduce carbonyl compounds in water, alcohol, or their mixture. The reduction is very exothermic and the reacting mixture is usually cooled to decrease the evaporation of solvent and reactants. Besides dissolving sodium borohydride and benzaldehyde, as representative carbonyl compound, the reaction in glycerol can be performed without cooling due to its high boiling temperature and thermal stability. A stoichiometric amount of sodium borohydride was added to benzaldehyde solution in glycerol and the yield of benzyl alcohol was $100 \%$ after 15 min (entry 2). Many other carbonyl compounds such as 1-phenylethanol, 1-octanone, and 2-octanone were also easily reduced in short times under similar conditions.

Catalysis plays an important role in the development of environmental friendly processes (Blaser 1999). It may lead to the replacement of toxic reagents and improvement of activity and selectivity that reduces formation of byproducts and thus leads to simpler, cleaner, and more effective separation processes. Heterogeneous catalysts have the distinct advantage that they can be easily separated and reused, while homogeneous catalysis is usually very active and selective. Heterogenization of TMCs in biphasic systems to combine the advantageous of homogeneous and heterogeneous catalysis is frequently used.

Catalytic reduction with molecular hydrogen is very common. The reduction of styrene, which has low solubility in glycerol, to ethylbenzene was chosen as representative reaction and showed high conversions with both homogeneous (1) and heterogeneous metal catalysts (entry 3). Using glycerol as reaction medium allowed also to recycle the complex. The conversion of the second catalytic cycle after extraction of ethylbenzene with diethyl ether and addition of fresh styrene to the glycerol and the TMC phase was equal to the extraction of the first cycle. Since styrene is poorly miscible in glycerol, addition of low amount of non-ionic surfactant, Pluronic (PE 6400 BASF), to the biphasic system yielded an emulsion system and thereby increased the reaction conversion. Thus glycerol can also offer non-aqueous biphasic and emulsion systems, which find many applications infields other than catalysis and organic synthesis.

Use of a polar solvent is also an advantage in the one phase palladium catalyzed Heck coupling, since it allows dissolving strong inorganic base, which activates the reaction (Alonso 2005). Performing the Heck coupling of iodobenzene and butyl acrylate with both homogeneous palladium complex $\mathbf{2}$ and supported palladium catalyst and with the addition of sodium carbonate yielded high yield of butyl cinnamate (entry 4). In this reaction, glycerol as $\mathrm{BmimPF}_{6}$ dissolved inorganic, organic, and organometallic compounds and allowed easy separation of the formed product by extraction.

Moreover, glycerol also tolerates the uses of microwave heating. Microwave heating has found many applications in organic synthesis, as it is cleaner and reduces reactions time substantially (Kappe 2004). As previously stated, besides solubility of reactants, a solvent is also utilized to transport heat. In microwave heating, the solvent selection is even more crucial. Microwave heating is based on the ability of a solvent to absorb microwave energy and convert it into heat. It is usually increases when the dielectric constant of the solvent rise and in the presence of hydroxyl groups. Hence, glycerol is a very attractive solvent due to its high polarity and high boiling point. As expected, the microwave-assisted Heck coupling of iodobenzene and butyl acrylate was faster under microwave irradiation than the reaction under conventional heating (entry 4).

Asymmetric catalysis is a powerful tool in the synthesis of fine and special chemicals. Though several chiral TMCs were successfully employed for this purpose, the use of biocatalysts has some advantages, since it proceeds in mild conditions and it does not require tedious complex synthesis. Biocatalysis is usually carried out in water, yet non-aqueous biocatalysis was also reported in organic solvent to overcome the low solubility of organics in water and to avoid side reactions. Lipase-catalyzed kinetic resolution of ester racemate is an example for such successful biocatalytic reaction. It involves the enantioselective transesterification (alcoholysis) of only one of the esters using an excess of alcohol as the resolving agent. The resolution of racemic mixture of 2-methyl heptanoate was hence performed with immobilized Candida antarctica lipase in glycerol (entry 6). It resulted in high alcohol yield and high enantioselectivities of the ester and the corresponding alcohol. In this reaction, glycerol as alcohol, also takes part as resolving agent and abstracts the formed acid.

Enantiopure compounds are also prepared by enantioselective transformation of prochiral compounds. This method increases the theoretical product yield to $100 \%$ and avoids complicated separation of the two enantiomers. For example, prochiral carbonyl compounds can be asymmetrically reduced with biocatalysts to the corresponding enantiomer. Several oxidoreductases are capable of efficiently reducing ketones in the combination of a cofactor such as NADPH or NADH, yet using the whole cell like baker's yeast (Saccharomyces cerevisiae) for chiral reduction is simpler, cheaper, and also attractive, since all of the cofactor is supplied by the microorganism. Water is the natural environment for baker's yeasts reductions and glucose or sucrose are usually employed as energy source and as electron donor in the regeneration of the cofactor. However, as previously 
mentioned, performing the reduction of prochiral ketones with baker's yeasts in water has several drawbacks: low solubility of the organic substrate, undesired side reactions such as hydrolysis, and difficult isolation of the product. Hence, the enantioselective reduction of various prochiral ketones was also studied in different organic solvents such as hexane, toluene, which destroys the cells and has severe environmental impact. Moreover, the low suspension of yeast in organic medium as well as the negligible solubility of glucose in these organic solvents leads to low reaction rates. Performing the reaction in glycerol allows dissolving ethyl acetoacetate and glucose and fine dispersion of the yeast, yielding high product yield and enantioselectivity (entry 5). Besides easy separation, immobilization of the yeast cells keeps some water around them and thus increases their performance.

\section{Conclusion}

To conclude, glycerol was successfully employed as versatile and alternative green solvent in variety of organic reactions and synthesis methodologies. High products conversions and selectivities were achieved. Besides solubility of the reactants and the catalysts and easy separation of the products, glycerol offers several other benefits such as TMCs recycling, microwave assisting reaction, and emulsion mode.

\section{References}

Alonso F, Beletskaya IP, Yus MM (2005) Non-conventional methodologies for transition-metal catalysed carbon-carbon coupling: a critical overview. Part 1. The Heck reaction. Tetrahedron 61:1177111835

Blaser HU, Studer M (1999) The role of catalysis for the clean production of fine chemicals. Appl Catal A Gen 189:191-204

Horváth IT, Rábai J (1994) Facile catalyst separation without water: fluorous biphase hydroformylation of olefins. Science 266:72-75

Howarth J, Jams P, Dai J (2001) Immobilized baker's yeast reduction of ketones in an ionic liquid, [bmim] $\mathrm{PF}_{6}$ and water mix. Tetrahedron Lett 42:7517-7519

Imhof A, Pine DJ (1997) Stability of nonaqueous emulsions. J Colloid Interface Sci 192:368-374

Jessop PG, Leitner W (1999) Chemical synthesis using supercritical fluids, Wiley, Weinheim

Kappe CO (2004) Controlled microwave heating in modern organic synthesis. Angew Chem Int Ed 43:6250-6284

March J (1992) Advanced organic chemistry: reactions, mechanisms, and structure, Wiley, New York

Reichardt C (1979) Solvent effects in organic chemistry. Verlag Chemie, Weinheim

Wasserscheid P, Welton T (2003) Ionic liquids in synthesis, Wiley, Weinheim 